\title{
Espectro de gotas de pulverização e controle da ferrugem-asiática-da-soja em cultivares com diferentes arquiteturas de planta
}

\author{
Mônica Paula Debortoli(1), Nédio Rodrigo Tormen ${ }^{(2)}$, Ricardo Silveiro Balardin(2), Diego Dalla Favera(2), \\ Marlon Tagliapietra Stefanello(2), Felipe Frigo Pinto(2) e Juliano Daniel Uebel(2)
}

\begin{abstract}
(1)Universidade Federal de Santa Maria (UFSM), Departamento de Engenharia Agrícola, Avenida Roraima, no 1.000, Camobi, CEP 97105-900 Santa Maria, RS. E-mail: mpdebortoli@yahoo.com.br (2)UFSM, Departamento de Defesa Fitossanitária. E-mail: nrtormen@yahoo.com.br, balardin@balardin.com, ddfavera@gmail.com, marlonstefanello@gmail.com, felipefrigo@hotmail.com, julianouebel_1@yahoo.com.br
\end{abstract}

Resumo - O objetivo deste trabalho foi determinar o espectro de gotas de pulverização ideal para o controle da ferrugem-asiática-da-soja (Phakopsora pachyrhizi), em cultivares com diferentes arquiteturas de planta. O experimento foi conduzido na safra de 2009/2010. A aplicação de fungicida (piraclostrobina + epoxiconazol, com óleo mineral) foi avaliada com quatro espectros de gotas de pulverização - muito fino, <119 $\mu \mathrm{m}$; fino, 119 a $216 \mu \mathrm{m}$; médio, 217 a $352 \mu \mathrm{m}$; e grosso, 353 a $464 \mu \mathrm{m}-$, em quatro cultivares de soja (BMX Apollo RR, NA 7636 RR, Fcep 53 RR e TMG 4001 RR) contrastantes quanto a índice de área foliar, estatura de plantas e número de ramos por planta. Foram quantificadas as variáveis: número de gotas por $\mathrm{cm}^{2}$, diâmetro mediano volumétrico das gotas, área abaixo da curva de progresso da ferrugem-asiática e produtividade da soja. A definição do espectro de gotas a ser utilizado deve considerar a cultivar e as condições ambientais em que a pulverização será realizada. A magnitude da proteção exercida pelo fungicida varia de acordo com a cobertura de plantas e a penetração de gotas no dossel, proporcionada pelos diferentes espectros de gota. O espectro de gotas fino proporciona boa deposição de gotas, controle da doença e produtividade, independentemente da cultivar avaliada.

Termos para indexação: Phakopsora pachyrhizi, cobertura de plantas, controle químico, deposição de gotas, penetração no dossel, tecnologia de aplicação.

\section{Spray droplet spectrum and control of Asian soybean rust in cultivars with different plant architecture}

\begin{abstract}
The objective of this work was to determine the ideal spray droplet spectrum for the control of Asian soybean rust (Phakopsora pachyrhizi), in cultivars with different plant architecture. The experiment was carried out in the 2009/2010 crop season. Fungicide application (pyraclostrobin + epoxyconazole, with mineral oil) was evaluated with four spray droplet spectra - very fine, $<119 \mu \mathrm{m}$; fine, 119 to $216 \mu \mathrm{m}$; medium, 217 to $352 \mu \mathrm{m}$; and coarse, 353 to $464 \mu \mathrm{m}$ - in four soybean cultivars (BMX Apollo RR, NA 7636 RR, Fcep $53 \mathrm{RR}$, and TMG $4001 \mathrm{RR}$ ) contrasting as to leaf area index, plant height, and number of branches per plant. The following variables were quantified: number of drops per $\mathrm{cm}^{2}$, median volume diameter of droplets, area under the progress curve of soybean rust, and soybean yield. The definition of the droplet spectrum to be used should consider cultivar and environmental conditions in which the pulverization will be done. The protection magnitude exerted by the fungicide varies according to the plant coverage and the penetration of droplets into the canopy provided by the different droplet spectra. The fine droplet spectrum provides good droplet deposition, disease control, and productivity, regardless of the evaluated cultivar.
\end{abstract}

Index terms: Phakopsora pachyrhizi, plant coverage, chemical control, droplet deposition, canopy penetration, application technology.

\section{Introdução}

A eficiência da tecnologia de aplicação é determinada pela distribuição adequada do produto no alvo. Segundo Paulsrud \& Montgomery (2005), a tecnologia de aplicação de fungicidas tem como objetivo produzir um tamanho de gotas que possibilite bom equilíbrio entre cobertura de plantas, penetração no dossel e deposição de gotas nas folhas. O sucesso no manejo da ferrugem-asiática-da-soja depende do monitoramento frequente da doença na área, da escolha do momento ideal de aplicação, do uso de fungicidas com maior

Pesq. agropec. bras., Brasília, v.47, n.7, p.920-927, jul. 2012 
residual, da boa cobertura do alvo pela aplicação e, em muitos casos, da reaplicação (Butzen et al., 2005a). De acordo com esse conceito, a tecnologia de aplicação, tanto a aérea como a terrestre, assume importante papel na eficácia de fungicidas para o manejo da ferrugem asiática (Butzen et al., 2005b).

A qualidade da aplicação depende de diversos fatores, como escolha de fungicidas eficientes, momento ideal para aplicação, frequência suficiente de aplicações, volume adequado de aplicação, tecnologia de aplicação ajustada ao alvo e à cultura (Cunha et al., 2010). Tanto a deposição quanto a perda de produtos são influenciadas por configurações de trabalho dos pulverizadores, velocidade do vento, estatura das plantas, condições meteorológicas, arquitetura de planta, características morfológicas - como pilosidade e cerosidade -, estádio de desenvolvimento e volume de aplicação (Souza et al., 2003).

A penetração de gotas no dossel de uma cultura é fator fundamental para o controle químico de doenças, especialmente das que iniciam o processo infeccioso nas folhas baixeiras, como é o caso da ferrugem-asiática-da-soja, causada pelo fungo Phakopsora pachyrhizi Syd. \& P. Syd. (Raetano, 2007). Assim, a variabilidade entre cultivares de soja, no que tange à arquitetura de plantas, impõe barreiras distintas à penetração de gotas no dossel no momento da aplicação. Essas barreiras precisam ser vencidas pelas gotas para que ocorra boa cobertura da planta pelo fungicida e, consequentemente, controle satisfatório da doença.

Apesar de existirem diversos trabalhos na área de tecnologia de aplicação que quantifiquem a deposição de calda no dossel de plantas de soja, poucos levam em consideração as barreiras impostas pelas diferenças de arquitetura entre as cultivares. Ross (1981) define arquitetura de planta como fatores que influenciam a forma, o tamanho, a geometria e a estrutura externa da planta. A arquitetura inclui diversos caracteres, como número de hastes e ramos, estrutura de hastes ou ramos (número e comprimento dos entrenós), e estrutura, tamanho e orientação de folhas (Huyghe, 1998).

A distribuição de gotas ao longo da planta depende da arquitetura de cada cultivar, já que as folhas da parte superior do dossel interceptam grande parte das gotas pulverizadas, o que impede que as folhas do terçoinferior recebam quantidade adequada de ingrediente ativo. Cada cultivar apresenta características peculiares no que diz respeito a sua arquitetura, que variam conforme a época de semeadura e as condições ambientais e de cultivo. Além de influenciar a deposição do fungicida, essas características criam condições de microclima no interior do dossel que podem favorecer ou não a ocorrência ou o rápido estabelecimento da doença (Wilson et al., 1999). Tem-se observado, também, tendência de as cultivares de soja apresentarem menor porte, maior potencial de ramificação e menor índice de área foliar, o que aumenta a necessidade de proteção dos tecidos para que as plantas possam expressar seu máximo potencial produtivo.

O objetivo deste trabalho foi determinar o espectro de gotas de pulverização ideal para o controle da ferrugem-asiática-da-soja, em cultivares com diferentes arquiteturas de planta.

\section{Material e Métodos}

O experimento foi conduzido na safra de 2009/2010, na área da Estação Experimental do Instituto Phytus, no Município de Itaara, RS $\left(29^{\circ} 58^{\prime} \mathrm{S}\right.$ e $53^{\circ} 81^{\prime} \mathrm{W}$, a $440 \mathrm{~m}$ de altitude). O espaçamento entre linhas utilizado foi de $0,5 \mathrm{~m}$, com 12 plantas por metro e população final de 240.000 plantas por hectare. A cultura foi estabelecida com plantio direto, em sucessão à cultura de aveia-preta (Avena strigosa Schreb).

Utilizou-se o delineamento experimental de blocos ao acaso, com quatro repetições, em arranjo fatorial 4x5: quatro cultivares de soja (BMX Apollo RR, NA 7636 RR, Fcep 53 RR e TMG 4001 RR), quatro espectros de gota - muito fino, <119 $\mu \mathrm{m}$; fino, 119 a $216 \mu \mathrm{m}$; médio, 217 a $352 \mu \mathrm{m}$; e grosso, 353 a $464 \mu \mathrm{m}$, segundo normas BCPC (British Crop Production Council) - e testemunha sem aplicação. A parcela experimental foi constituída de seis linhas de semeadura de $5 \mathrm{~m}$ de comprimento $\left(15 \mathrm{~m}^{2}\right)$, com área útil de $8 \mathrm{~m}^{2}$. A área útil da parcela experimental foi obtida tendo-se desconsiderado as linhas de semeadura das laterais e $0,5 \mathrm{~m}$ em cada cabeceira da parcela.

A primeira aplicação do fungicida - piraclostrobina + epoxiconazol $\left(66,5+25 \mathrm{~g} \mathrm{ha}^{-1}\right)+$ Assist (óleo mineral) $\left(0,33 \% \mathrm{v} \mathrm{v}^{-1}\right)$ - foi efetuada no fechamento das entrelinhas de plantio, seguida de mais duas aplicações aos 21 dias após a primeira aplicação e aos 14 dias após a segunda aplicação. Utilizou-se pulverizador costal pressurizado a $\mathrm{CO}_{2}$ e volume de aplicação de $150 \mathrm{~L} \mathrm{ha}^{-1}$. O espectro de gotas foi obtido a partir da 
escolha da ponta de pulverização, do ajuste da pressão de trabalho e da velocidade de caminhamento, sem alteração no volume de aplicação.

A calibração do espectro de gotas das diferentes combinações de ponta de pulverização com pressão de trabalho foi realizada antes da aplicação, a partir da coleta de cartões hidrossensíveis e da determinação do diâmetro mediano volumétrico (DMV). Os cartões também foram utilizados para a coleta de dados logo após as aplicações do produto. Esses cartões são confeccionados a partir do tratamento de papel brilhante com tintura sensível à água, o azul de bromofenol. Este corante é amarelo, quando seco, e o contato com as gotas d'água produz manchas azuis (Turner \& Huntington, 1970; Matthews, 2000).

A disposição dos cartões no interior do dossel foi realizada por meio do emprego de haste metálica, que foi posicionada perpendicularmente à linha da cultura. A haste apresentava três suportes onde os cartões foram fixados. A posição de cada cartão nos terços foi ajustada à estatura de cada cultivar (terços inferior, médio e superior do dossel).

Logo após a aplicação dos tratamentos, os cartões foram rapidamente coletados e acondicionados em papel adesivo para serem digitalizados em "scanner" com resolução de varredura de 1.200 dpi. A partir da análise da imagem digital dos cartões, realizada com aplicativo CIR 1.5 (Inta, 2002), foram obtidos a cobertura e a penetração das gotas de pulverização, com base no número de gotas por $\mathrm{cm}^{2}$ e no DMV em cada terço.

A arquitetura das plantas foi avaliada por meio da determinação da estatura de plantas, do número de ramos por planta e do índice de área foliar (IAF) no momento das aplicações. A estatura foi medida em campo, com régua de madeira, e representou a distância compreendida entre o colo da planta e o último ponto de crescimento da haste principal. O número de ramos foi obtido por meio de contagem direta, logo após a remoção das folhas, tendo-se considerado como ramo as ramificações com, no mínimo, dois nós. Para a determinação do IAF, as plantas foram coletadas e tiveram todas as folhas destacadas e dispostas junto de régua graduada, sobre superfície, para serem fotografadas com câmera digital posicionada a $1,5 \mathrm{~m}$ acima das folhas. As fotos foram submetidas à análise de área foliar por meio do programa Quant v1.0.2 (Vale et al., 2003).
A severidade de ferrugem asiática foi avaliada mediante determinação do percentual de área foliar com sintomas da doença. Essa avaliação foi realizada visualmente em cada parcela, e representou a média do percentual de tecido com sintomas, em todas as plantas analisadas. As avaliações foram realizadas aos 7, 14, 21 e 28 dias após a última aplicação dos tratamentos. As notas de severidade da doença serviram para o cálculo da área abaixo da curva de progresso (AACP) da doença. Quando as plantas atingiram estádio R8, a colheita das plantas foi realizada, para a determinação da produtividade final da soja.

Os dados foram submetidos ao teste Shapiro-Wilk, que mostrou a normalidade dos dados. A significância dos fatores foi determinada pela análise de variância, por meio do programa estatístico Assistat 7.5 Beta (Silva \& Azevedo, 2002). As médias foram comparadas pelo teste de Tukey, a 5\% de probabilidade. Os dados obtidos não necessitaram ser transformados para a realização das análises.

\section{Resultados e Discussão}

Observou-se interação entre as cultivares e os espectros de gota avaliados, para as variáveis relacionadas à deposição de gotas (numero de gotas por $\mathrm{cm}^{2}$ e DMV). Nas três aplicações, foi verificada redução no DMV do terço superior para o inferior (Tabela 1). Bretthauer et al. (2008), ao avaliar espectro de gotas médio e muito grosso para controle de ferrugem-asiática-da-soja, relataram que o DMV na porção inferior das plantas diminuía, em média, 12,6 e $11,6 \%$, respectivamente.

Para Yu et al. (2009), o tamanho de gotas é um dos parâmetros mais importantes para o controle eficaz de pragas e doenças. Portanto, o espectro de gotas deve ter diâmetro mediano volumétrico suficiente para depositar no alvo, sem evaporar, a calda de pulverização com o produto. Contudo, o espectro também deve proporcionar boa cobertura do alvo pelo ingrediente ativo (Reichard et al., 1977). Para isso, é necessário que a tecnologia de aplicação produza gotas mais finas.

Na primeira aplicação, houve maior uniformidade na distribuição de gotas no interior do dossel do que na segunda e na terceira (Tabela 2). Nessa aplicação, foram observadas diferenças significativas na deposição de gotas, de acordo com o espectro testado. 
Tabela 1. Diâmetro mediano volumétrico obtido nas três aplicações com diferentes espectros de gota, em quatro cultivares de soja ${ }^{(1)}$.

\begin{tabular}{|c|c|c|c|c|c|}
\hline \multirow{2}{*}{ Cultivar } & \multicolumn{4}{|c|}{ Espectro de gotas } & \multirow[t]{2}{*}{ Média } \\
\hline & Muito fino & Fino & Médio & Grosso & \\
\hline & \multicolumn{5}{|c|}{ Primeira aplicação, terço superior } \\
\hline BMX Apollo RR & $109,3 \mathrm{aC}^{1}$ & $286,2 \mathrm{aB}$ & $417,0 \mathrm{aA}$ & $412,8 \mathrm{abA}$ & \multirow{4}{*}{$305,7 \mathrm{a}$} \\
\hline NA 7636 RR & $109,4 \mathrm{aC}$ & $290,8 \mathrm{aB}$ & $406,7 \mathrm{aA}$ & $366,4 \mathrm{bA}$ & \\
\hline Fcep 53 RR & $134,9 \mathrm{aD}$ & $238,5 \mathrm{aC}$ & $337,0 \mathrm{bB}$ & $470,5 \mathrm{aA}$ & \\
\hline \multirow[t]{2}{*}{ TMG $4001 \mathrm{RR}$} & $122,6 \mathrm{aD}$ & $298,1 \mathrm{aC}$ & $417,6 \mathrm{aB}$ & $472,7 \mathrm{aA}$ & \\
\hline & \multicolumn{5}{|c|}{ Terço médio } \\
\hline BMX Apollo RR & $119,2 \mathrm{aB}$ & $241,9 \mathrm{aA}$ & $241,9 \mathrm{bA}$ & $270,7 \mathrm{bA}$ & \multirow{4}{*}{$234,8 b$} \\
\hline NA 7636 RR & $119,9 \mathrm{aC}$ & $242,7 \mathrm{aB}$ & $364,4 \mathrm{aA}$ & $391,8 \mathrm{aA}$ & \\
\hline Fcep 53 RR & $137,9 \mathrm{aB}$ & $172,2 \mathrm{aB}$ & $341,2 \mathrm{aA}$ & $266,3 \mathrm{bA}$ & \\
\hline \multirow{2}{*}{ TMG $4001 \mathrm{RR}$} & $133,3 \mathrm{aB}$ & $203,6 \mathrm{aB}$ & $188,3 \mathrm{bB}$ & $322,0 \mathrm{abA}$ & \\
\hline & \multicolumn{5}{|c|}{ Terço inferior } \\
\hline BMX Apollo RR & $126,0 \mathrm{aB}$ & $191,4 \mathrm{aB}$ & $181,3 \mathrm{cB}$ & $290,6 \mathrm{bA}$ & \multirow{4}{*}{$207,8 \mathrm{c}$} \\
\hline NA 7636 RR & $110,7 \mathrm{aC}$ & $158,5 \mathrm{abBC}$ & $199,3 \mathrm{bcB}$ & $395,3 \mathrm{aA}$ & \\
\hline Fcep 53 RR & $71,8 \mathrm{aC}$ & $157,4 \mathrm{abB}$ & $259,1 \mathrm{abA}$ & $270,9 \mathrm{bA}$ & \\
\hline TMG $4001 \mathrm{RR}$ & $135,5 \mathrm{aC}$ & $109,4 \mathrm{bC}$ & 277,9 & $390,2 \mathrm{aA}$ & \\
\hline Média & $119,2 \mathrm{D}$ & $215,9 \mathrm{C}$ & $302,6 \mathrm{~B}$ & $360,0 \mathrm{~A}$ & \\
\hline \multirow[t]{2}{*}{ CV (\%) } & \multicolumn{4}{|c|}{12,53} & \\
\hline & \multicolumn{4}{|c|}{ Segunda aplicação, terço superior } & \multirow{5}{*}{$327,8 \mathrm{a}$} \\
\hline BMX Apollo RR & $126,3 \mathrm{aB}^{1}$ & $276,5 \mathrm{aAB}$ & $423,8 \mathrm{aA}$ & $392,1 \mathrm{aA}$ & \\
\hline NA 7636 RR & $121,4 \mathrm{aC}$ & $254,2 \mathrm{aBC}$ & $449,1 \mathrm{aAB}$ & $499,3 \mathrm{aA}$ & \\
\hline Fcep 53 RR & $123,2 \mathrm{aB}$ & $313,4 \mathrm{aAB}$ & $442,3 \mathrm{aA}$ & $492,5 \mathrm{aA}$ & \\
\hline \multirow[t]{2}{*}{ TMG $4001 \mathrm{RR}$} & $123,1 \mathrm{aB}$ & $271,4 \mathrm{aAB}$ & $482,7 \mathrm{aA}$ & $453,0 \mathrm{aA}$ & \\
\hline & \multicolumn{4}{|c|}{ Terço médio } & \multirow{5}{*}{$215,2 b$} \\
\hline BMX Apollo RR & $132,0 \mathrm{aB}$ & $194,1 \mathrm{abB}$ & $218,9 \mathrm{aAB}$ & $292,2 \mathrm{aA}$ & \\
\hline NA 7636 RR & $122,6 \mathrm{aC}$ & $157,4 \mathrm{bBC}$ & $237,9 \mathrm{aB}$ & $354,4 \mathrm{aA}$ & \\
\hline Fcep 53 RR & $146,6 \mathrm{aB}$ & $154,6 \mathrm{bB}$ & $258,3 \mathrm{aA}$ & $286,3 \mathrm{aA}$ & \\
\hline \multirow[t]{2}{*}{ TMG $4001 \mathrm{RR}$} & $105,9 \mathrm{aB}$ & $252,3 \mathrm{aA}$ & $238,9 \mathrm{aA}$ & $290,2 \mathrm{aA}$ & \\
\hline & \multicolumn{4}{|c|}{ Terço inferior } & \\
\hline BMX Apollo RR & $101,2 \mathrm{aB}$ & $148,3 \mathrm{abB}$ & $265,8 \mathrm{aA}$ & $331,6 a A$ & \\
\hline NA 7636 RR & $106,2 \mathrm{aB}$ & $92,6 \mathrm{bB}$ & $225,1 \mathrm{aA}$ & $289,3 \mathrm{aA}$ & \\
\hline Fcep 53 RR & $112,6 \mathrm{aC}$ & $187,7 \mathrm{aBC}$ & $212,8 \mathrm{aAB}$ & $298,6 \mathrm{aA}$ & $199,1 \mathrm{c}$ \\
\hline TMG $4001 \mathrm{RR}$ & $93,5 \mathrm{aC}$ & $193,0 \mathrm{aB}$ & $226,6 \mathrm{aAB}$ & $301,5 \mathrm{aA}$ & \\
\hline Média & $117,9 \mathrm{C}$ & $208,0 \mathrm{~B}$ & $306,9 \mathrm{~A}$ & $356,7 \mathrm{~A}$ & \\
\hline CV (\%) & & 20 & 09 & & \\
\hline & & Terceira apl & icação, terço & superior & \\
\hline BMX Apollo RR & $127,6 \mathrm{aB}^{1}$ & $200,9 \mathrm{aB}$ & $376,7 \mathrm{aA}$ & $440,3 \mathrm{aA}$ & \\
\hline NA 7636 RR & $122,7 \mathrm{aC}$ & $247,8 \mathrm{aBC}$ & $350,1 \mathrm{aAB}$ & $475,9 \mathrm{aA}$ & \\
\hline Fcep 53 RR & $139,7 \mathrm{aB}$ & $259,3 \mathrm{aB}$ & $289,7 \mathrm{aB}$ & $483,6 \mathrm{aA}$ & $293,4 \mathrm{a}$ \\
\hline TMG $4001 \mathrm{RR}$ & $114,2 \mathrm{aC}$ & $239,7 \mathrm{aBC}$ & $357,5 \mathrm{aAB}$ & $468,4 \mathrm{aA}$ & \\
\hline & & & erço médio & & \\
\hline BMX Apollo RR & $125,0 \mathrm{aB}$ & $245,6 \mathrm{aAB}$ & $214,7 \mathrm{aAB}$ & $333,1 \mathrm{aA}$ & \\
\hline NA 7636 RR & $115,3 \mathrm{aB}$ & $201,5 \mathrm{aB}$ & $220,0 \mathrm{aAB}$ & $362,2 \mathrm{aA}$ & \\
\hline Fcep 53 RR & $92,4 \mathrm{aB}$ & $207,9 \mathrm{aAB}$ & $245,6 \mathrm{aA}$ & $306,8 \mathrm{aA}$ & $220,1 \mathrm{~b}$ \\
\hline TMG $4001 \mathrm{RR}$ & $124,9 \mathrm{aB}$ & $225,8 \mathrm{aAB}$ & $273,1 \mathrm{aAB}$ & $323,3 \mathrm{aA}$ & \\
\hline & & & rço inferior & & \\
\hline BMX Apollo RR & $113,8 \mathrm{aB}$ & $175,9 \mathrm{aB}$ & $148,6 \mathrm{aB}$ & $314,5 \mathrm{aA}$ & \\
\hline NA 7636 RR & $122,5 \mathrm{aB}$ & $202,4 \mathrm{aAB}$ & $205,2 \mathrm{aAB}$ & $284,8 \mathrm{aA}$ & \\
\hline Fcep 53 RR & $110,7 \mathrm{aB}$ & $164,1 \mathrm{aAB}$ & $217,6 \mathrm{aAB}$ & $246,2 \mathrm{aA}$ & $193,4 \mathrm{~b}$ \\
\hline TMG $4001 \mathrm{RR}$ & $102,6 \mathrm{aB}$ & $169,5 \mathrm{aB}$ & $148,3 \mathrm{aB}$ & $367,0 \mathrm{aA}$ & \\
\hline Média & $117,6 \mathrm{D}$ & $211,7 \mathrm{C}$ & $253,9 \mathrm{~B}$ & $367,2 \mathrm{~A}$ & \\
\hline $\mathrm{CV}(\%)$ & & 22 & 87 & & \\
\hline
\end{tabular}

Tabela 2. Cobertura de gotas (número de gotas por $\mathrm{cm}^{2}$ ) obtida nas três aplicações com diferentes espectros de gota, em quatro cultivares de soja ${ }^{(1)}$.

\begin{tabular}{|c|c|c|c|c|c|}
\hline \multirow{2}{*}{ Cultivar } & \multicolumn{4}{|c|}{ Espectro de gotas } & \multirow[t]{2}{*}{ Média } \\
\hline & Muito fino & Fino & Médio & Grosso & \\
\hline & \multicolumn{5}{|c|}{ Primeira aplicação, terço superior } \\
\hline BMX Apollo RR & $238,7 \mathrm{bAB}$ & ${ }^{12} 248,0 \mathrm{abA}$ & $189,0 \mathrm{abB}$ & $191,0 \mathrm{aB}$ & \multirow{4}{*}{$210,4 \mathrm{a}$} \\
\hline NA 7636 RR & $357,0 \mathrm{aA}$ & $221,3 \mathrm{abB}$ & $140,3 \mathrm{bcC}$ & $137,7 \mathrm{bC}$ & \\
\hline Fcep 53 RR & $231,7 \mathrm{bAB}$ & $268,7 \mathrm{aA}$ & $217,7 \mathrm{aB}$ & $212,3 \mathrm{aB}$ & \\
\hline \multirow[t]{2}{*}{ TMG $4001 \mathrm{RR}$} & $247,7 \mathrm{bA}$ & $198,7 \mathrm{bA}$ & $134,3 \mathrm{cB}$ & $131,7 \mathrm{bB}$ & \\
\hline & \multicolumn{5}{|c|}{ Terço médio } \\
\hline BMX Apollo RR & $131,3 \mathrm{aA}$ & $106,7 \mathrm{aA}$ & $43,3 \mathrm{aB}$ & $50,3 \mathrm{aB}$ & \multirow{4}{*}{$75,6 \mathrm{~b}$} \\
\hline NA 7636 RR & $80,3 \mathrm{bcA}$ & $78,3 \mathrm{aAB}$ & $37,3 \mathrm{aB}$ & $48,0 \mathrm{aAB}$ & \\
\hline Fcep 53 RR & $78,3 \mathrm{cA}$ & $95,7 \mathrm{aA}$ & $53,7 \mathrm{aA}$ & $56,3 \mathrm{aA}$ & \\
\hline \multirow[t]{2}{*}{ TMG $4001 \mathrm{RR}$} & $121,7 \mathrm{abA}$ & $112,7 \mathrm{aAB}$ & $45,0 \mathrm{aC}$ & $71,3 \mathrm{aBC}$ & \\
\hline & \multicolumn{5}{|c|}{ Terço inferior } \\
\hline BMX Apollo RR & $65,0 \mathrm{aA}$ & $33,7 \mathrm{aAB}$ & $24,3 \mathrm{aAB}$ & $22,0 \mathrm{aB}$ & \multirow{4}{*}{$33,0 \mathrm{c}$} \\
\hline NA 7636 RR & $25,3 \mathrm{aA}$ & $32,0 \mathrm{aA}$ & $12,3 \mathrm{aA}$ & $15,0 \mathrm{aA}$ & \\
\hline Fcep 53 RR & $42,3 \mathrm{aA}$ & $44,0 \mathrm{aA}$ & $36,3 \mathrm{aA}$ & $27,7 \mathrm{aA}$ & \\
\hline TMG $4001 \mathrm{RR}$ & $69,0 \mathrm{aA}$ & $40,3 \mathrm{aAB}$ & $20,0 \mathrm{aB}$ & $19,3 \mathrm{aB}$ & \\
\hline Média & $140,7 \mathrm{~A}$ & $123,3 \mathrm{~A}$ & $79,5 \mathrm{~B}$ & $81,9 \mathrm{~B}$ & \\
\hline CV (\%) & \multicolumn{4}{|c|}{12,49} & \\
\hline
\end{tabular}

\begin{tabular}{|c|c|c|c|c|c|}
\hline \multirow{5}{*}{$\begin{array}{l}\text { BMX Apollo RR } \\
\text { NA } 7636 \text { RR } \\
\text { Fcep } 53 \text { RR } \\
\text { TMG 4001 RR }\end{array}$} & \multicolumn{5}{|c|}{ Segunda aplicação, terço superior } \\
\hline & $253,0 \mathrm{aA}^{1}$ & $241,7 \mathrm{aAB}$ & $160,7 \mathrm{aB}$ & $168,3 \mathrm{aAB}$ & \multirow{4}{*}{$192,5 \mathrm{a}$} \\
\hline & $205,3 \mathrm{aA}$ & $133,0 \mathrm{bA}$ & $171,0 \mathrm{aA}$ & $163,7 \mathrm{aA}$ & \\
\hline & $266,7 \mathrm{aA}$ & $216,0 \mathrm{abAB}$ & $188,7 \mathrm{aAB}$ & $144,7 \mathrm{aB}$ & \\
\hline & $277,3 \mathrm{aA}$ & $237,0 \mathrm{aA}$ & $150,3 \mathrm{aB}$ & $102,0 \mathrm{aB}$ & \\
\hline & \multicolumn{5}{|c|}{ Terço médio } \\
\hline ollo RR & $79,3 \mathrm{aA}$ & $51,3 \mathrm{aB}$ & $44,7 \mathrm{aB}$ & $25,7 \mathrm{abC}$ & \multirow{4}{*}{$38,2 b$} \\
\hline NA 7636 RR & $42,0 \mathrm{cAB}$ & $57,3 \mathrm{aA}$ & $35,7 \mathrm{abB}$ & $27,0 \mathrm{aB}$ & \\
\hline Fcep 53 RR & $55,3 \mathrm{bcA}$ & $30,3 \mathrm{bB}$ & $28,7 \mathrm{bcB}$ & $11,7 \mathrm{bC}$ & \\
\hline \multirow[t]{2}{*}{ TMG $4001 \mathrm{RR}$} & $65,7 \mathrm{abA}$ & $23,0 \mathrm{bB}$ & $15,7 \mathrm{cB}$ & $18,0 \mathrm{abB}$ & \\
\hline & \multicolumn{5}{|c|}{ Terço inferior } \\
\hline BMX Apollo RR & $22,7 \mathrm{aA}$ & $14,7 \mathrm{abB}$ & $9,3 \mathrm{aBC}$ & $5,3 \mathrm{aC}$ & \multirow{4}{*}{$13,3 \mathrm{c}$} \\
\hline NA 76 & $20,7 \mathrm{aA}$ & $17,3 \mathrm{aAB}$ & $11,7 \mathrm{aBC}$ & $8,0 \mathrm{aC}$ & \\
\hline Fcep 53 RR & $25,7 \mathrm{aA}$ & $10,3 \mathrm{bB}$ & $6,7 \mathrm{aB}$ & $6,0 \mathrm{aB}$ & \\
\hline TMG $4001 \mathrm{RR}$ & $19,3 \mathrm{aA}$ & $14,7 \mathrm{abA}$ & $13 \mathrm{aAB}$ & $6,7 \mathrm{aB}$ & \\
\hline Média & $111,1 \mathrm{~A}$ & $87,2 \mathrm{~B}$ & $69,7 \mathrm{C}$ & $57,3 \mathrm{C}$ & \\
\hline \multirow{2}{*}{$\mathrm{CV}(\%)$} & \multicolumn{4}{|c|}{20,80} & \\
\hline & \multicolumn{5}{|c|}{ Terceira aplicação, terço superior } \\
\hline BMX Apollo RR & $267,7 \mathrm{aA}^{1}$ & $219,3 \mathrm{aAB}$ & $166,0 \mathrm{aBC}$ & $136,3 \mathrm{aC}$ & \multirow{4}{*}{$207,8 \mathrm{a}$} \\
\hline NA 76 & $303,7 \mathrm{aA}$ & $203,0 \mathrm{aB}$ & $144,0 \mathrm{aB}$ & $137,7 \mathrm{aB}$ & \\
\hline Fcep 53 RR & $272,0 \mathrm{aA}$ & $250,7 \mathrm{aA}$ & $205,7 \mathrm{aAB}$ & $149,3 \mathrm{aB}$ & \\
\hline \multirow[t]{2}{*}{ TMG $4001 \mathrm{RR}$} & $257,3 \mathrm{aA}$ & $223,3 \mathrm{aA}$ & $209,0 \mathrm{aA}$ & $179,7 \mathrm{aA}$ & \\
\hline & \multicolumn{5}{|c|}{ Terço médio } \\
\hline BMX Apollo RR & $108,0 \mathrm{aA}$ & $46,3 \mathrm{bcB}$ & $39,7 \mathrm{aB}$ & $36,0 \mathrm{aB}$ & \multirow{4}{*}{$46,7 b$} \\
\hline NA $7636 \mathrm{RR}$ & $66,7 \mathrm{bA}$ & $31,0 \mathrm{cB}$ & $16,0 \mathrm{bB}$ & $17,0 \mathrm{aB}$ & \\
\hline Fcep 53 RR & $59,7 \mathrm{bB}$ & $86,3 \mathrm{aA}$ & $45,0 \mathrm{aBC}$ & $28,3 \mathrm{aC}$ & \\
\hline \multirow[t]{2}{*}{ TMG $4001 \mathrm{RR}$} & $79,7 \mathrm{bA}$ & $51,7 \mathrm{bB}$ & $17,3 \mathrm{bC}$ & $18,0 \mathrm{aC}$ & \\
\hline & \multicolumn{5}{|c|}{ Terço inferior } \\
\hline BMX Apollo RR & $26,3 \mathrm{aA}$ & $22,0 \mathrm{aA}$ & $13,0 \mathrm{aB}$ & $13,0 \mathrm{aB}$ & \multirow{4}{*}{$14,6 \mathrm{c}$} \\
\hline NA $7636 \mathrm{RR}$ & $17,7 \mathrm{bA}$ & $14,3 \mathrm{aAB}$ & $9,0 \mathrm{aB}$ & $8,0 \mathrm{aB}$ & \\
\hline Fcep $53 \mathrm{RR}$ & $21,7 \mathrm{abA}$ & $18,7 \mathrm{aAB}$ & $11,7 \mathrm{aB}$ & $11,7 \mathrm{aB}$ & \\
\hline TMG $4001 \mathrm{RR}$ & $16,3 \mathrm{bA}$ & $14,7 \mathrm{aAB}$ & $10,0 \mathrm{aAB}$ & $6,3 \mathrm{aB}$ & \\
\hline Média & $124,7 \mathrm{~A}$ & $98,4 \mathrm{~B}$ & $73,9 \mathrm{C}$ & $61,8 \mathrm{C}$ & \\
\hline CV (\%) & & & & & \\
\hline
\end{tabular}

${ }^{(1)}$ Médias seguidas de letras iguais, maiúsculas na linha (entre espectros) e minúsculas na coluna (entre cultivares), não diferem pelo teste de Tukey, a $5 \%$ de probabilidade. 
A deposição no terço superior, nas três aplicações, foi superior a 100 gotas por $\mathrm{cm}^{2}$, tendo chegado a atingir valores acima de 250 gotas por $\mathrm{cm}^{2}$, apropriados para aplicação de fungicidas (Ozeki \& Kunz, 1998; Christofoletti, 1999).

Em geral, espectros de gotas muito finos e finos proporcionaram maior deposição de gotas que espectros médios e grossos (Tabela 2). As cultivares TMG 4001 RR e BMX Apollo RR apresentaram redução significativa na deposição de gotas no terço inferior à medida que o espectro mudou de muito fino para grosso. Cunha et al. (2010) verificaram que gotas produzidas por pontas de pulverização com dispositivo antideriva (gotas com DMV maior) penetraram menos no dossel da soja que gotas produzidas pelas mesmas pontas de pulverização sem este dispositivo (com menor DMV).

Wolf \& Daggupati (2009), ao avaliar a penetração de gotas no dossel de soja propiciada por 20 diferentes pontas de pulverização, com espectros de gotas variados (fino, médio e grosso), obtiveram cobertura no terço inferior que não ultrapassou $10 \%$ do tecido. Para esses autores, a densidade de folhas é o fator que mais influencia a penetração das gotas.

Nas três aplicações e em todos os espectros de gotas avaliados, o terço superior apresentou o maior número de gotas por $\mathrm{cm}^{2}$ (Tabela 2), com deposição de dose de ingrediente ativo suficiente para proteção adequada das folhas. Segundo Zhu et al. (2002), à medida que as plantas crescem, aumenta a concentração das gotas no topo do dossel, e a deposição de gotas frequentemente é maior que a obtida nos terços inferior e médio. Houve redução de 20 a 30\% no número de gotas depositadas com espectro grosso, em comparação ao espectro muito fino.

$\mathrm{Na}$ segunda aplicação, as cultivares BMX Apollo RR e NA 7636 RR apresentaram maior deposição de gotas no terço médio, com espectro fino, médio e grosso (Tabela 2). Na terceira aplicação, no entanto, o comportamento das cultivares mudou ligeiramente, e a cultivar Fcep 53 RR substituiu a NA 7636 RR entre as com os maiores valores de deposição de gotas médias e grossas, no terço médio.

Com base nos dados de deposição das três aplicações, a cultivar BMX Apollo RR foi a menos afetada pela alteração no espectro, e as cultivares TMG 4001 RR e NA 7636 RR foram as mais sensíveis à alteração.

As quatro cultivares avaliadas apresentaram características de arquitetura de planta distintas
(Tabela 3). A estatura de plantas apresentou diferenças significativas entre as quatro cultivares, nas três aplicações, e a cultivar TMG 4001 RR foi a de maior porte, enquanto as cultivares BMX Apollo RR e Fcep 53 RR, em geral, foram as de menor. Para número de ramos por planta e IAF, essas cultivares figuraram, comumente, entre as que apresentaram os menores valores, diferentemente da cultivar TMG $4001 \mathrm{RR}$, que, em geral, teve maior potencial de emissão de ramos e maior IAF. A cultivar NA 7636 RR apresentou valores intermediários, semelhantes, muitas vezes, ao das cultivares BMX Apollo RR e Fcep 53 RR.

A aplicação de fungicida proporcionou redução significativa da AACP da ferrugem asiática em todas as cultivares avaliadas (Tabela 4). O comportamento das cultivares BMX Apollo RR e Fcep 53 RR se destacou nos espectros médio e grosso. Essas cultivares raramente diferiram quanto aos parâmetros de deposição de gotas e aos de arquitetura de plantas (Tabelas 1, 2 e 3).

As cultivares NA 7636 RR e TMG 4001 RR apresentaram maior variação no controle da doença proporcionado pelos diferentes espectros de gota (Tabela 4). Com gotas muito finas e finas, elas apresentaram AACP semelhante ao das demais, mas maiores valores nos outros espectros. Essas

Tabela 3. Estatura de planta, número de ramos por planta e índice de área foliar das quatro cultivares de soja avaliadas, no momento de cada aplicação de fungicida ${ }^{(1)}$.

\begin{tabular}{lccc}
\hline Cultivar & $\begin{array}{c}\text { Primeira } \\
\text { aplicação }\end{array}$ & $\begin{array}{c}\text { Segunda } \\
\text { aplicação }\end{array}$ & $\begin{array}{c}\text { Terceira } \\
\text { aplicação }\end{array}$ \\
\hline BMX Apollo RR & $65,5 \mathrm{c}$ & $\begin{array}{c}\text { Estatura de plantas }(\mathrm{cm}) \\
76,3 \mathrm{c}\end{array}$ & $78,3 \mathrm{bc}$ \\
NA 7636 RR & $75,0 \mathrm{~b}$ & $82,3 \mathrm{~b}$ & $83,0 \mathrm{~b}$ \\
Fcep 53 RR & $70,8 \mathrm{~b}$ & $76,0 \mathrm{c}$ & $75,0 \mathrm{c}$ \\
TMG 4001 RR & $97,5 \mathrm{a}$ & $102,3 \mathrm{a}$ & $102,3 \mathrm{a}$ \\
\hline CV (\%) & 2,72 & 2,77 & 2,91 \\
\hline & & Número de ramos por planta \\
BMX Apollo RR & $2,8 \mathrm{~b}$ & $3,0 \mathrm{c}$ & $3,0 \mathrm{c}$ \\
NA 7636 RR & $3,9 \mathrm{~b}$ & $5,1 \mathrm{~b}$ & $5,1 \mathrm{~b}$ \\
Fcep 53 RR & $2,5 \mathrm{~b}$ & $3,4 \mathrm{bc}$ & $3,1 \mathrm{c}$ \\
TMG 4001 RR & $6,9 \mathrm{a}$ & $7,4 \mathrm{a}$ & $7,5 \mathrm{a}$ \\
\hline CV (\%) & 18,40 & 17,33 & 17,87 \\
\hline & & Índice de área foliar & \\
BMX Apollo RR & $3,7 \mathrm{a}{ }^{1}$ & $4,0 \mathrm{~b}$ & $2,8 \mathrm{~b}$ \\
NA 7636 RR & $3,7 \mathrm{a}$ & $3,6 \mathrm{~b}$ & $3,5 \mathrm{~b}$ \\
Fcep 53 RR & $3,6 \mathrm{a}$ & $3,3 \mathrm{~b}$ & $3,3 \mathrm{~b}$ \\
TMG 4001 RR & $4,3 \mathrm{a}$ & $6,6 \mathrm{a}$ & $6,0 \mathrm{a}$ \\
\hline CV (\%) & 17,49 & 13,90 & 18,74 \\
\hline
\end{tabular}

(1)Médias seguidas de letras iguais, na coluna, não diferem pelo teste de Tukey, a 5\% de probabilidade. 
cultivares também apresentaram as maiores variações de deposição de gotas entre espectros, tiveram maior potencial de ramificação e maior IAF (Tabelas 1, 2 e 3). Portanto, houve interferência dos componentes de arquitetura de plantas na qualidade de aplicação.

Não houve diferença significativa no controle da doença quando da mudança do espectro de muito fino para fino, em nenhuma das cultivares (Tabela 4). Porém, em todas as aplicações, o espectro de gotas muito fino proporcionou cobertura de gotas significativamente superior à dos demais espectros (Tabela 2). Esse resultado é indicativo de que, apesar do número de gotas ter sido maior com espectro muito fino, não houve incremento de controle em comparação ao espectro fino. Quando a gota é aplicada em direção ao alvo, ela pode ser perdida por deriva ou por evaporação (Bukovac et al., 1995). Esses processos variam conforme o tamanho da gota, a estrutura da superfície foliar, as propriedades físicas da formulação e as condições ambientais. Em muitos casos, gotas de fungicidas sistêmicos precisam permanecer em contato com a folha (alvo) por um período mínimo, para que a planta possa absorver o ingrediente ativo (Yu et al., 2009). Segundo Matthews (2000), gotas pequenas têm tempo de vida (tempo de extinção) muito reduzido. Assim, se o ingrediente ativo perder seu diluente, há formação de uma partícula minúscula de ingrediente concentrado que não é absorvido e que pode ser removido da superfície foliar.

Yu et al. (2009), ao comparar gotas de diferentes DMV, em condições extremas de umidade relativa do ar (URA), verificaram que gotas com DMV de $246 \mu \mathrm{m}$ evaporam dez vezes mais rápido que gotas com DMV

Tabela 4. Área abaixo da curva de progresso da ferrugem-asiática-da-soja após a aplicação de fungicida com diferentes espectros de gotas, em quatro cultivares de $\operatorname{soja} a^{(1)}$.

\begin{tabular}{lcccc}
\hline Espectro & BMX Apollo RR & NA 7636 RR & Fcep 53 RR TMG 4001 RR \\
\hline Testemunha & $336,0 \mathrm{dA}$ & $387,8 \mathrm{cA}$ & $574,8 \mathrm{bA}$ & $656,3 \mathrm{aA}$ \\
Muito fino & $3,8 \mathrm{aB}$ & $15,4 \mathrm{aC}$ & $11,5 \mathrm{aB}$ & $17,49 \mathrm{aC}$ \\
Fino & $5,3 \mathrm{aB}$ & $12,6 \mathrm{aC}$ & $10,6 \mathrm{aB}$ & $18,6 \mathrm{aC}$ \\
Médio & $6,9 \mathrm{cB}$ & $25,0 \mathrm{abBC}$ & $14,4 \mathrm{bcB}$ & $32,2 \mathrm{aBC}$ \\
Grosso & $13,0 \mathrm{bB}$ & $36,8 \mathrm{aB}$ & $19,1 \mathrm{bB}$ & $42,5 \mathrm{aB}$ \\
\hline CV $(\%)$ & \multicolumn{5}{c}{7,65} \\
\hline
\end{tabular}

${ }^{(1)}$ Médias seguidas de letras iguais, maiúsculas na coluna (entre espectros) e minúsculas na linha (entre cultivares), não diferem pelo teste de Tukey, a $5 \%$ de probabilidade. de $800 \mu \mathrm{m}$, a $60 \%$ de URA. Além do menor número de gotas obtido no interior do dossel (terços inferior e médio), em comparação ao terço superior, também foi observada redução significativa no DMV das gotas que atingiram o interior do dossel. Esses fatores podem ajudar a explicar a ausência de melhoria no controle da doença com espectro de gotas muito fino, que, apesar de ter maior número de gotas por $\mathrm{cm}^{2}$, não diferiu do espectro de gotas fino.

Isso pode justificar, também, o fato de o espectro de gotas grossas ter possibilitado, em algumas cultivares, controle da doença comparável ao proporcionado pela aplicação de gotas finas (Tabela 4). A retenção e a absorção do ingrediente ativo na superfície foliar são dependentes da cobertura de gotas e do tempo que a gota permanece no alvo (Brazee et al., 2004). No entanto, o maior tempo de permanência da gota sobre a superfície foliar, obtida com gotas grossas, em geral, não compensa a menor cobertura que esse espectro de gotas possibilita.

Os dados indicam que, para fins de controle da ferrugem asiática nas cultivares BMX Apollo RR e Fcep 53 RR, o resultado da aplicação deve ser satisfatório independentemente do espectro de gotas utilizado. Já nas cultivares NA 7636 RR e TMG 4001 RR, gotas de espectro muito fino, fino e médio poderão reduzir significativamente os níveis da doença.

As variações no controle da doença, propiciadas pelos diferentes espectros de gotas, impactaram significativamente a produtividade das cultivares avaliadas (Tabela 5). O impacto positivo do controle da doença possibilitou incrementos de produtividade que variaram de 11,6 a 40,6\%, em comparação à testemunha. A cultivar NA $7636 \mathrm{RR}$ foi a mais produtiva na ausência de aplicação de fungicida, e a cultivar TMG 4001 RR foi a mais sensível à doença. A cultivar Fcep 53 RR apresentou bom desempenho produtivo, com produção de até $3.884,7 \mathrm{~kg} \mathrm{ha}^{-1}$.

A aplicação com gotas finas propiciou os maiores incrementos de produtividade nas quatro cultivares, tendo sempre diferido significativamente da testemunha. Esse espectro, portanto, apresenta possibilidade de ampla utilização, em cultivares de distintas arquiteturas de planta. Para Butzen et al. (2005b), gotas finas/médias com DMV de $220 \mu \mathrm{m}$ ou menores têm proporcionado a melhor cobertura na aplicação para controle da ferrugem asiática. Ozkan 
Tabela 5. Desempenho produtivo da soja $\left(\mathrm{kg} \mathrm{ha}^{-1}\right)$ e variação na produtividade $(\Delta)$ em comparação à testemunha, após aplicação de fungicida com diferentes espectros de gota, em quatro cultivares de soja.

\begin{tabular}{|c|c|c|c|c|c|c|c|c|}
\hline \multirow{2}{*}{ Espectro } & \multicolumn{2}{|c|}{ BMX Apollo RR } & \multicolumn{2}{|c|}{ NA 7636 RR } & \multicolumn{2}{|c|}{ Fcep 53 RR } & \multicolumn{2}{|c|}{ TMG $4001 \mathrm{RR}$} \\
\hline & Produtividade & $\Delta(\%)$ & Produtividade & $\Delta(\%)$ & Produtividade & $\Delta(\%)$ & Produtividade & $\Delta(\%)$ \\
\hline Testemunha & $2829,7 \mathrm{Bd}$ & 0,0 & $3135,3 \mathrm{aC}$ & 0,0 & $2763,9 \mathrm{bC}$ & 0,0 & $2159,4 \mathrm{cC}$ & 0,0 \\
\hline Muito fino & $3590,6 \mathrm{aAB}$ & 26,9 & $3696,2 \mathrm{aA}$ & 17,9 & $3607,7 \mathrm{aB}$ & 30,5 & $2896,9 \mathrm{bA}$ & 34,2 \\
\hline Fino & $3717,2 \mathrm{aA}$ & 31,4 & $3763,0 \mathrm{aA}$ & 20,0 & $3884,7 \mathrm{aA}$ & 40,6 & $3029,7 \mathrm{bA}$ & 40,3 \\
\hline Médio & $3332,8 \mathrm{aC}$ & 17,8 & $3453,0 \mathrm{aB}$ & 10,1 & $3480,4 \mathrm{aB}$ & 25,9 & $2429,7 \mathrm{bB}$ & 12,5 \\
\hline Grosso & $3434,4 \mathrm{abBC}$ & 21,4 & $3655,7 \mathrm{aAB}$ & 16,6 & $3413,3 \mathrm{bB}$ & 23,5 & $2410,9 \mathrm{cB}$ & 11,6 \\
\hline CV (\%) & & & & & & & & \\
\hline
\end{tabular}

${ }^{(1)}$ Médias seguidas de letras iguais, maiúsculas na coluna (entre espectros) e minúsculas na linha (entre cultivares), não diferem pelo teste de Tukey, a $5 \%$ de probabilidade.

et al. (2007) também apontam que esse espectro de gotas proporciona maior cobertura e penetração de gotas no dossel da soja.

As cultivares com maior ramificação e IAF - NA7636 RR e TMG 4001 RR - apresentaram maior variação na produtividade, em função dos diferentes espectros avaliados (Tabelas 3 e 5). As cultivares BMX Apollo RR e Fcep 53 RR tiveram variações muito pequenas de produtividade. Esses resultados são indicativos de que cultivares com maior capacidade de ramificação e maior IAF necessitam de maior cobertura de gotas. Nesse caso, o espectro de gotas fino apresentou número de gotas suficiente para proteger os tecidos, ao contrário dos espectros médio e grosso. Provavelmente, para atingir maior cobertura de gotas nessas cultivares, com espectros médio e grosso, o volume de aplicação deverá ser aumentado. Segundo Butzen et al. (2005b) e Ozkan et al. (2007), em maiores densidades de folhas, o aumento de volume pode fornecer cobertura e penetração suficientes para proteger o tecido foliar da soja contra o patógeno.

\section{Conclusões}

1. A definição do espectro de gotas para aplicação de calda fungicida deve considerar a cultivar e as condições ambientais na qual a operação será realizada.

2. O espectro de gotas fino (DMV, 119 a $216 \mu \mathrm{m}$ ) proporciona bons resultados em termos de deposição de gotas, controle da doença e produtividade, nas quatro cultivares de soja.

3. A magnitude da proteção da soja à ferrugem asiática varia em função da cobertura e da penetração de gotas proporcionadas pela aplicação.

\section{Referências}

BRAZEE, R.D.; BUKOVAC, M.J.; ZHU, H. Diffusion model for plant cuticular penetration by spray-applied weak organic acid bioregulator in presence or absence of ammonium nitrate. Transactions of the ASAE, v.47, p.629-635, 2004.

BRETTHAUER, S.M.; MUELLER, T.A.; DERKSEN, R.C.; ZHU, H.; BODE, L.E. The effects of spray application rate and droplet size on applications to control soybean rust. St. Joseph: American Society of Agricultural and Biological Engineers, 2008. 9p. (ASABE. Paper, 084219).

BUKOVAC, M.J.; LEON, J.M.; COOPER, J.A.; WHITMOYER, R.E.; REICHARD, D.L.; BRAZEE, R.D. Spray droplet: plant surface interaction and deposit formation as related to surfactants and spray volume. In: INTERNATIONAL SYMPOSIUM ON ADJUVANTS FOR AGROCHEMICALS, 4., 1995, Melbourne. Proceedings. Melbourne: ISSA, 1995. p.177-185.

BUTZEN, S.; BEUDOT, F.; MCINNES, B. Asian soybean rust: fungicides. Crop Insights, v.15, p.1-5, 2005a. Available at: <https://www.pioneer.com/ home/site/us/agronomy/library/template.CONTENT/ guid.6604153B-DB01-CE9E-6A9D-B916EE2573BB>. Accessed on: 1 Aug. 2012.

BUTZEN, S.; MARCON, A.; MCINNES, B.; SCHUH, W. Asian soybean rust: fungicide application technology. Crop Insights, v.15, p.1-6, 2005b. Available at: <https:/www.pioneer.com/pv obj_cache/pv_obj_id 1EAD36E2D0ACC79A055D5437DEDB2 9BFBC8A0600/filename/sr_apptech.pdf >. Accessed on: 1 Aug. 2012.

CHRISTOFOLETTI, J.C. Pulverização ou aplicação? São Paulo: Teejet South América, 1999. 71p. (Boletim técnico, BT-01/99).

CUNHA, J.P.A.R. da; COELHO, L.; ARAÚJO, R.G.C. Spray nozzle and adjuvant effects on fungicidal control of soybean Asian rust. Interciência, v.35, p.765-768, 2010.

HUYGHE, C. Genetics and genetic modifications of plant architecture in grain legumes: a review. Agronomie, v.18, p.383-411, 1998 . 
INTA. T\&C CIR: conteo y tipificación de impactos de pulverización. Versión 1.5. Buenos Aires: INTA, 2002.

MATTHEWS, G.A. Pesticide application methods. $3^{\text {rd }}$ ed. Berkshire: International Pesticide Application Research Centre, 2000. 432p.

OZEKI, Y.; KUNZ, R.P. Tecnologia de aplicação aérea: aspectos práticos. In: GUEDES, J.V.C.; DORNELLES, S.H.B. Tecnologia e segurança na aplicação de agrotóxicos: novas tecnologias. Santa Maria: UFSM, 1998. p.65-78.

OZKAN, E.; BRETTHAUER, S.; MILES, M.; WOLF, R. Application basics. In: DORRANCE, A.E.; DRAPER, M.A.; HERSHMAN, D.E. (Ed.). Using foliar fungicides to manage soybean rust. Columbus: The Ohio State University, 2007. p.61-70.

PAULSRUD, B.E.; MONTGOMERY, M. Characteristics of fungicides used in field crops. Champaign: University of Illinois at Urbana, 2005. 18p. (Report on plant disease, 1002).

RAETANO, C.G. Assistência de ar e outros métodos de aplicação a baixo volume em culturas de baixo fuste: a soja como modelo. Summa Phytopathologica, v.33, p.105-106, 2007. Suplemento.

REICHARD, D.L.; RETZER, H.J.; LILJEDAHL, L.A.; HALL, F.R. Spray droplet size distributions delivered by air blast orchard sprayers. Transactions of the ASABE, v.20, p.232-237, 1977.

ROSS, J. The radiation regime and architecture of plant stands. Dordrecht: Kluwer, 1981. 391p.

SILVA, F. de A.S. e; AZEVEDO, C.A.V. de. Versão do programa computacional Assistat para o sistema operacional Windows. Revista Brasileira de Produtos Agroindustriais, v.4, p.71-78, 2002.
SOUZA, R.T.; PALLADIN, L.A.; VELINI, E.D.; KAMINSKI, V.; BRAUN, S. Pulverização eletrostática. Cultivar, n. 21, p.1-10, 2003. (Caderno técnico, máquinas).

TURNER, C.R.; HUNTINGTON, K.A. The use of water sensitive dye for the detection and assessment of small spray droplets. Journal of Agricultural Engineering Research, v.15, p.385-387, 1970.

VALE, F.X.R.; FERNADES FILHO, E.I.; LIBERATO, J.R. QUANT: a software for plant disease severity assessment. In: INTERNATIONAL CONGRESS OF PLANT PATHOLOGY, 8., 2003, Christchurch. Proceedings. Christchurch: International Society for Plant Pathology, 2003. p.105.

WILSON, P.A.; HANAN, J.S.; ROOM, P.M.; CHAKRABORTY, S.; DOLEY, D. Using Lindenmayer systems to model morphogenesis in a tropical pasture legume Stylosanthes scabra. Canadian Journal of Botany, v.77, p.394-403, 1999.

WOLF, R.E.; DAGGUPATI, N.P. Nozzle type effect on soybean canopy penetration. Applied Engineering in Agriculture, v.25, p.23-30, 2009.

YU, Y.; ZHU, H.; OZKAN, H.E.; DERKSEN, R.C.; KRAUSE, C.R. Evaporation and deposition coverage area of droplets containing insecticides and spray additives on hydrophilic, hydrophobic, and crabapple leaf surfaces. Transactions of the ASAE, v.52, p.39-49, 2009.

ZHU, H.; ROWLAND, D.L.; DORNER, J.W.; DERKSEN, R.C.; SORENSEN, R.B. Influence of plant structure, orifice size, and nozzle inclination on spray penetration into peanut canopy. Transactions of the ASAE, v.45, p.1295-1301, 2002. 